\title{
DNA methylation and expression analyses reveal epialleles for the foliar disease resistance genes in peanut (Arachis hypogaea L.)
}

\author{
R. S. Bhat ${ }^{1 *} \mathbb{D}$, J. Rockey ${ }^{2}$, Kenta Shirasawa ${ }^{3}$, I. S. Tilak ${ }^{1 \dagger}$, M. P. Brijesh Patil ${ }^{1 \dagger}$ and V. B. Reddy Lachagari ${ }^{4}$
}

\begin{abstract}
Objective: Low DNA sequence polymorphism despite enormous phenotypic variations in peanut indicates the possible role of epigenetic variations. An attempt was made to analyze genome-wide DNA methylation pattern and its influence on gene expression across 11 diverse genotypes of peanut.

Results: The genotypes were subjected to bisulfite sequencing after 21 days of sowing (DAS). CHG regions showed the highest $(30,537,376)$ DNA methylation followed by $\mathrm{CpG}(30,356,066)$ and $\mathrm{CHH}(15,993,361)$ across 11 genotypes. The B sub-genome exhibited higher DNA methylation sites $(46,294,063)$ than the A sub-genome $(30,415,166)$. Overall, the DNA methylation was more frequent in inter-genic regions than in the genic regions. The genes showing altered methylation and expression between the parent (TMV 2) and its EMS-derived mutant (TMV 2-NLM) were identified. Foliar disease resistant genotypes showed significant differential DNA methylation at 766 sites corresponding to 25 genes. Of them, two genes (Arahy.1XYC2X on chromosome 01 and Arahy.00Z2SH on chromosome 17) coding for senescence-associated protein showed differential expression with resistant genotypes recording higher fragments per kilobase of transcript per million mapped reads (FPKM) at their epialleles. Overall, the study indicated the variation in the DNA methylation pattern among the diverse genotypes of peanut and its influence of gene expression.
\end{abstract}

Keywords: Peanut, Genotypes differing for foliar disease response, DNA methylome, Transcriptome, Differentially methylated genes, Expression levels of methylated genes

\section{Introduction}

Peanut (Arachis hypogaea L. $2 \mathrm{n}=4 \mathrm{x}=40$ ) is an important legume food and oilseed crop world-wide. The breeding efforts mainly focus on productivity, disease and insect resistance, oil quality etc. Genomics-assisted breeding has been successfully employed $[1,2]$ with the development of the genomic resources including the genome sequence of the cultivated allotetraploid peanut $[3,4]$. Apart from the genome, the epigenome also influences the gene function and the phenotype [5]. Despite

\footnotetext{
*Correspondence: bhatrs@uasd.in

${ }^{\dagger}$ I. S. Tilak and M. P. Brijesh Patil contributed equally to this work

1 Department of Biotechnology, University of Agricultural Sciences, Dharwad 580 005, India

Full list of author information is available at the end of the article
}

the narrow genetic base, peanut shows enormous phenotypic variability, which probably hints at the possible role of epigenetic variations in altering the phenotype.

DNA methylation along with histone modifications and chromatin changes generally make up the epigenome. In plants, genome-wide DNA methylation analyses have indicated the modification of epigenome with drought [6], growth and development, hybridization [7, 8], induced and spontaneous mutations [9-15] etc. The pattern of DNA methylation within the genome of Arabidopsis [16], rice [9], wheat [17], maize [18], Brassica juncea [6], Plantago lagopus [19] etc. has been uncovered, and its interdependency on transcription was demonstrated. The stability and heritability of the epigenetic variations are being characterized [20]. 
Though the putative genes coding for cytosine- 5 DNA methyltransferase (C5-MTases) and demethylase mediating the DNA methylation pattern have been identified in the progenitors of peanut [21], the pattern of DNA methylation in the cultivated peanut is yet to be analyzed. In this study, an effort was made to analyze the DNA methylome and its influence on transcriptome of the peanut genotypes contrasting for the productivity traits and the response to foliar diseases with an objective of identifying the epialleles contributing for the desirable phenotype in peanut so that an efficient breeding programme can be devised for such traits.

\section{Main text Methods}

A total of 11 genotypes (Table 1) were employed for methylome sequencing. Seeds of these genotypes were sown in the pots and the seedlings were grown in the greenhouse with optimal conditions. Leaf samples were collected for DNA and RNA isolation from a single 21-day old seedling. DNA was isolated using Qiagen DNeasy Plant Mini Kit (Cat \# 69104) and the RNA was isolated using Qiagen RNeasy Plant Mini Kit (Cat \# 74904). Bisulfite treatment was done using Zymo EZ DNA Methylation-Gold Kit. DNA methylome library was constructed using illumine TruSeq ${ }^{\circledR}$ DNA Methylation Kit. RNA library was constructed using Illumina TruSeq ${ }^{\circledR}$ Stranded mRNA Kit. The quality of the libraries was checked using TapeStation and Qubit. DNA sequencing was carried out using Illumina Hiseq 2500, and the RNA sequencing was carried out using Illumina Hiseq 2500 and Illumina Hiseq 4000 with two technical replicates and without any biological replicates.

\section{Methyl-Seq analysis}

Raw fastq files were pre-processed using AdapterRemoval v2 [22] tool. Using bwa-meth [23] program, the preprocessed reads were aligned with the Arachis hypogaea reference genome downloaded from PeanutBase [24]. The genomic sites showing DNA methylation were identified using MethylDackel program. Differential methylation was analyzed using methylKit [25] $\mathrm{R}$ package. The DNA methylation pattern was compared across the genotypes at $q$-value cutoff 0.01 and methylation percentage change cutoff 25 using methylKit.

\section{RNA-Seq analysis}

Raw data pre-processing was done using AdapterRemoval v2 [22] tool. The pre-processed reads were aligned with silva database using bowtie2 [26] to remove any ribosomal RNA. The reference genome of the Arachis hypogaea was downloaded from PeanutBase [24] and employed for alignment using STAR aligner [27] to get the alignment bam files. Differential expression analysis was executed using cuffdiff program of cufflinks package [28]. $\log 2$ fold change cutoff \pm 2 and $P$-value cutoff 0.05 were used for identifying differentially expressed genes. Custom scripts were developed to identify genes that were differentially methylated as well as differentially expressed.

\section{qRT-PCR}

Expression of the selected genes were confirmed using qRT-PCR. Total RNA was isolated from the young leaves on 21 DAS using Qiagen Rneasy mini kit (Qiagen, USA), and the quantity was checked using NanoDrop spectrophotometer (ND-2000, Thermo fisher scientific, USA). cDNA was synthesized using Affinity Script qPCR cDNA

Table 1 Genotypes used for DNA methylome and transcriptome sequencing

\begin{tabular}{|c|c|c|c|}
\hline S. no. & Genotypes & Pedigree & Features \\
\hline 1 & GPBD 4 & KRG $1 \times$ CS $16($ ICGV 86855) & $\begin{array}{l}\text { Early maturing with high pod growth rate, has high oil content and } \\
\text { resistant to LLS and rust }\end{array}$ \\
\hline 2 & VG 9514 & Derivative of $A$. cardenasii and cv. CO 1 & Rust resistant \\
\hline 3 & ICGV 86855 & Interspecific derivative (A hypogaea x A. cardenasii) & Resistant to LLS, rust and drought \\
\hline 4 & ICGV 86699 & Derivative of $[$ A. hypogaea $\times($ A. batizocoi $\times$ A. duranensis $)]$ & Multiple resistance to ELS, LLS, rust, bud necrosis, stem and pod rots \\
\hline 5 & ICGV 99005 & Derivative of $[$ A. hypogaea $\times(A$. batizocoi $\times$ A. duranensis $)]$ & Immune or with high resistance to LLS and rust \\
\hline 6 & TAG 24 & Selection from TGS-2 (TG-18A × M13) × TGE-1 & $\begin{array}{l}\text { Early maturity, high harvest index (50-55\%) and susceptible to LLS and } \\
\text { rust }\end{array}$ \\
\hline 7 & TMV 2 & Selection from 'Gudhiyatham bunch' & Early maturity, wide adaptability and susceptible to LLS and rust \\
\hline 8 & $J 24$ & Selection from 'EC-9493' & $\begin{array}{l}\text { Early maturity, high yielding short duration and susceptible to LLS and } \\
\text { rust }\end{array}$ \\
\hline 9 & DER & Dh $3-20 \times C G C-1$ & Runner type and susceptible to LLS \\
\hline 10 & $\mathrm{VL} 1$ & Mutant of DER & Rust resistant \\
\hline 11 & TMV 2-NLM & Mutant of TMV 2 & Drought tolerant and resistant to LLS and rust \\
\hline
\end{tabular}




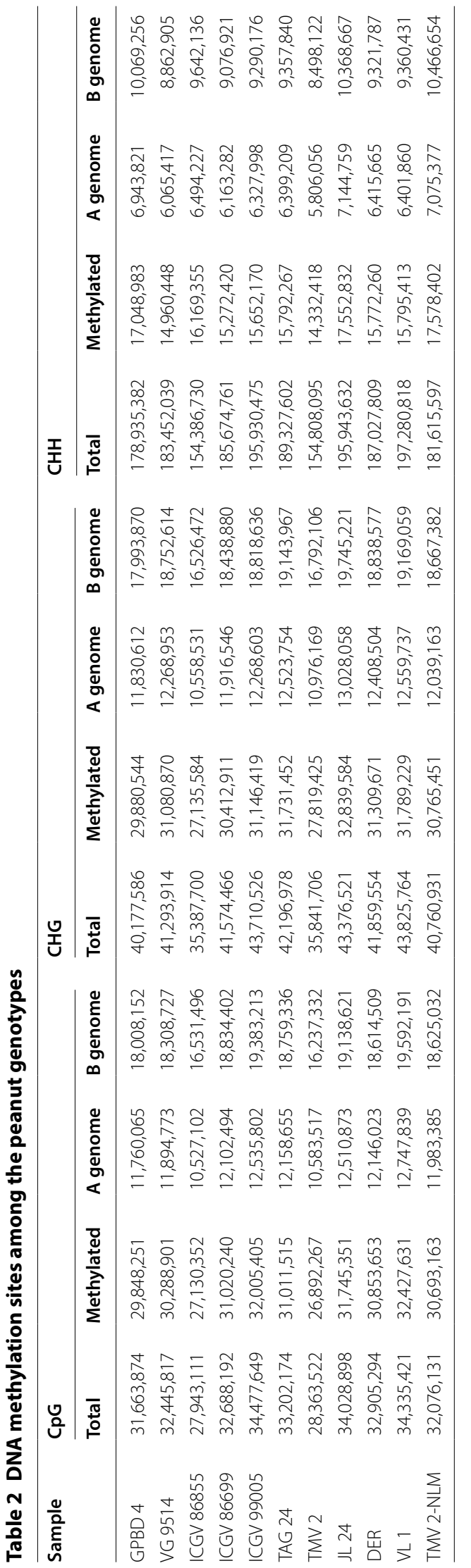


synthesis kit (Agilent Technologies, USA). qPCR assay was carried out to check the expression levels of selected genes using SYBR Green chemistry (Brilliant II SYBR Green qPCR master mix (Agilent Technologies, USA) using two technical replicates. Level of fold change over a house-keeping gene [glucose-6-phosphate 1-dehydrogenase (G6PD); Arahy.XC1VLW on chromosome 1/Arahy.74FNJK on chromosome 11] was worked out for each sample using multiple samples as biological replicates. The fold change across the samples were compared using the $t$ test.

\section{Results and discussion}

On an average, 127,852,977 bisulphite sequencing reads were generated for each sample (Additional file 1: Table S1). As high as $99.96 \%$ reads mapped on to the genome of cultivated peanut. Only VG 9514 had relatively low mapped reads, indicating its divergence from the cultivated peanut probably due to the contribution from $A$. cardenasii. The number of mapped reads at each DNA methylated site ranged from 1 to 1658 (Additional file 2: Table S2). Among the 11 genotypes, 75,973,928 sites belonged to the category where all the mapped reads (100\%) showed cytosine methylation (Additional file 3: Table S3). Similarly, 101,137,805 sites belonged to the category where at least $50 \%$ of the mapped reads showed cytosine methylation. The number of sites where less than $50 \%$ of the mapped reads showed cytosine methylation was $126,487,183$.

On an average, $255,319,879$ plausible DNA methylation sites were found among the 11 genotypes, of them $76,886,803$ sites showed DNA methylation with $100 \%$ reads showing methylation (Table 2 ). The $B$ sub-genome exhibited higher DNA methylation sites $(46,294,063)$ than the A sub-genome $(30,415,166)$ across the genotypes. A total of 177,574 sites were found in the scaffolds. CHG (where $\mathrm{H}$ is $\mathrm{A}, \mathrm{C}$ or $\mathrm{T}$ ) region showed the highest methylation sites $(30,537,376)$ regions, followed by CpG $(30,356,066)$ and $\mathrm{CHH}(15,993,361)$ regions across the genotypes. This observation is in line with the previous reports $[29,30]$ that the DNA methylation in plants is found both in $\mathrm{CpG}$ and non-CpG (CHG and $\mathrm{CHH}$, where $\mathrm{H}$ is $\mathrm{A}, \mathrm{C}$ or $\mathrm{T}$ ) contexts in contrast to mammals where DNA methylation occurs predominantly at CpG dinucleotides.

Among the 11 genotypes, JL 24 and TMV 2 showed the highest $(82,137,767)$ and the lowest methylation sites $(69,044,110)$, respectively (Table 3$)$. Such a natural epigenetic variation was also observed among the different ecotypes of Arabidopsis [31]. Many of the sites were found to be conserved across for DNA methylation across the genotypes of peanut. A total of 5,379,101 sites showed DNA methylation across all the 11 genotypes.
The sites showing genotype-specific DNA methylation ranged from 6,575,363 (TMV 2) to 9,190,780 (JL 24) (Table 3).

On an average, inter-genic regions (70,464,637 sites) were more prone for DNA methylation than the genic regions including $2 \mathrm{~kb}$ upstream and $2 \mathrm{~kb}$ downstream regions $(6,422,166$ sites) (Table 3$)$. Within the genic regions, the introns $(1,590,263)$ showed a greater number of DNA methylation sites than the exonic regions $(971,274)$. The $2 \mathrm{~kb}$ upstream and $2 \mathrm{~kb}$ downstream regions had 3,860,629 DNA methylation sites, indicating higher proportion of DNA methylation at the upstream and downstream regions than the gene body region. The distribution of DNA methylation within the genome especially in the promoter and gene body regions is very important as it influences the gene expression [32].

Of the 67,124 genes (31,359 in A genome, 35,110 in B genome and 655 on scaffolds) in peanut, the number of genes showing at least one methylated site ranged from 51,179 (ICGV 86855) to 55,497 (ICGV 99005) (Table 3). Of them, Arahy.ODU9MH, a 342,359 bp long gene on chromosome 11, showed the highest number of methylated sites, which ranged from 11,488 (ICGV 86855) to 14,026 (JL 24). Within Arahy.ODU9MH, the promoter region had 131 methylated sites, while the gene body (142 in exons and 12,573 in introns) had 12,715 sites. The expression (FPKM) of the 53,740 genes varied widely among the 11 genotypes (Additional file 4: Table S4). Arahy.ODU9MH with the highest DNA methylations sites did not show any expression at 21 DAS in the leaves of the 11 genotypes. Fifty genes with wide range of FPKM across the genotypes were selected and checked for the DNA methylation. Arahy.FHUH7B on chromosome 10 showing the highest FPKM of 54,951 had a maximum of 102 DNA methylation sites (Additional file 5: Table S5). Many of these genes showed negative association between the number of DNA methylation sites and FPKM among the genotypes.

Fourteen C5-MTase coding genes and ten DNA demethylase coding genes identified in the diploid peanut earlier [21] were analysed for DNA methylation and expression. A considerable variation was observed for methylation across the genes, however, not much variation was observed for methylation across the genotypes (Additional file 6: Table S6). A DME-like A gene Arahy. R549UJ (Aradu.4D5YM) of 15,531 bp length on chromosome 8 showed the highest number of methylation (as high as 586). Forty-four sites were found in the promoter region, while 542 sites were in the gene body (48 in exon and 494 in intron). This gene did not show any expression at 21 DAS in the leaves of the 11 genotypes.

An attempt was made to enumerate the differentially methylated sites between a parent (TMV 2) and 
Table 3 DNA methylation pattern among the peanut genotypes

\begin{tabular}{llllllllll}
\hline Sample & $\begin{array}{l}\text { Total } \\
\text { methylated } \\
\text { sites }\end{array}$ & Genic & Exonic & Intronic & $\begin{array}{l}\text { Upstream } \\
\mathbf{2} \text { kb) }\end{array}$ & $\begin{array}{l}\text { Downstream } \\
\mathbf{( 2 ~ k b )}\end{array}$ & Intergenic & Unique sites $\begin{array}{l}\text { Genes } \\
\text { with methylation }\end{array}$ \\
\hline GPBD 4 & $76,777,778$ & $2,542,609$ & 954,981 & $1,615,686$ & $2,422,857$ & $2,002,208$ & $74,235,169$ & $8,252,512$ & 53,177 \\
VG 9514 & $76,330,219$ & $2,491,420$ & 926,732 & $1,591,061$ & $2,353,495$ & $1,938,882$ & $73,838,799$ & $7,557,697$ & 53,344 \\
ICGV 86855 & $70,435,291$ & $2,236,778$ & 832,360 & $1,427,817$ & $2,197,445$ & $1,793,923$ & $68,198,513$ & $7,549,158$ & 51,179 \\
ICGV 86699 & $76,705,571$ & $2,608,306$ & $1,006,455$ & $1,631,016$ & $2,396,086$ & $1,994,004$ & $74,097,265$ & $7,856,020$ & 54,699 \\
ICGV 99005 & $78,803,994$ & $2,745,542$ & $1,068,392$ & $1,708,747$ & $2,508,886$ & $2,096,784$ & $76,058,452$ & $8,283,176$ & 55,497 \\
TAG 24 & $78,535,234$ & $2,591,128$ & 972,282 & $1,647,757$ & $2,459,092$ & $2,043,738$ & $75,944,106$ & $8,207,597$ & 53,838 \\
TMV 2 & $69,044,110$ & $2,237,309$ & 853,885 & $1,407,484$ & $2,143,795$ & $1,756,481$ & $66,806,801$ & $6,575,363$ & 51,866 \\
JL 24 & $82,137,767$ & $2,755,372$ & $1,042,721$ & $1,743,612$ & $2,574,365$ & $2,137,468$ & $79,382,395$ & $9,190,780$ & 54,645 \\
DER & $77,935,584$ & $2,555,262$ & 964,170 & $1,618,932$ & $2,394,118$ & $1,984,129$ & $75,380,322$ & $7,889,130$ & 53,555 \\
VL 1 & $80,012,273$ & $2,737,311$ & $1,045,365$ & $1,723,338$ & $2,540,239$ & $2,105,390$ & $77,274,962$ & $8,693,213$ & 54,876 \\
TMV 2-NLM & $79,037,016$ & $2,675,872$ & $1,018,559$ & $1,687,698$ & $2,533,141$ & $2,103,929$ & $76,361,144$ & $9,031,971$ & 54,463 \\
\hline
\end{tabular}

its EMS-derived mutant (TMV 2-NLM). The two genotypes significantly differed for 650 methylation sites, of which 240 and 401 were found in the A and B genome (remaining nine on the scaffolds), respectively. Again, the inter-genic region showed a greater number of DNA methylated sites (605) than the genic regions $(45 ; 23$ in exons and 22 in introns). Thirty-seven genes exhibited differential methylation, of which eight showed differential expression (Additional file 7: Table S7a), indicating the influence of EMS mutagenesis on DNA methylation.

In an attempt to identify the differentially DNA methylated sites, foliar disease resistant (GPBD 4, VG 9514, ICGV 86855, ICGV 99005 and ICGV 86699) and susceptible (TAG 24, TMV 2 and JL 24) groups of genotypes were constructed. The common sites within susceptible group were compared with the common sites within the resistant group. In total, 766 sites showed significantly differential DNA methylation. Of these, 331 sites were in the A genome and 433 sites were in the B genome. In total, 731 methylation sites were in the inter-genic regions and 35 were in the genic regions (19 in exons and 16 in introns). Interestingly, four differentially DNA methylated sites $(1,001,785,1,001,813,1,021,671$ and $1,305,680$ ) mapped to the QTL region (for LLS) on A02 and one $(134,350,159)$ mapped to the QTL region (for rust) on A03 [33]. Of these sites, only one $(1,305,680)$ was in a genic (Arahy.42YDET) region. However, this gene has not been regarded as a candidate gene for foliar disease resistance [33]. Based on the genomic position of the DNA methylation sites, 25 genes were found to be differentially methylated $(q \leq 0.01)$ between resistant and susceptible genotypes. Of these genes, two genes (Arahy.1XYC2X on chromosome 01 and Arahy.00Z2SH on chromosome 17) coding for senescence-associated protein showed differential expression with resistant genotypes recording higher FPKM values (Additional file 7: Table S7b). It was interesting to note the methylation pattern within Arahy. $1 X Y C 2 X$ differed between the resistant and susceptible groups, indicating the epialleles at this locus. The candidate genes identified for late leaf spot (four genes) and rust (six genes) resistance in the previous study [33] did not show any DNA methylation, indicating that breeding for foliar disease resistance can depend only on the genetic variation. FPKM values for the transcripts at these loci were on par between the resistant and the susceptible genotypes. This was also confirmed by the qRT-PCR where some of these genes showed non-significant fold changes between the two groups (Additional file 8: Table S8).

\section{Limitations}

A detailed genome-wide DNA methylation pattern was reported from the 11 diverse genotypes of peanut for the first time by analyzing the leaf samples collected at 21 DAS. Also, the influence of induced mutagenesis on the pattern of DNA methylation was assessed. Differentially methylated sites were identified between the foliar disease resistant and susceptible genotypes. The genes showing differential methylation and expression were identified. However, these data need to be validated by comparing the samples collected at different stages of growth and development and varying conditions (diseased and normal) to identify the role of DNA methylation, and its influence on gene expression in peanut. 


\section{Supplementary information}

Supplementary information accompanies this paper at https://doi. org/10.1186/s13104-020-4883-y.

Additional file 1: Table S1. Features of DNA methylome reads generated in this study.

Additional file 2: Table S2. Read depth observed 100\% Freq C among the $\mathrm{CPG}, \mathrm{CHG}$ and $\mathrm{CHH}$ regions among the 11 genotypes of peanut.

Additional file 3: Table S3. Number of reads with various Freq $C$ values at $\mathrm{CPG}, \mathrm{CHG}$ and $\mathrm{CHH}$ regions among the 11 genotypes of peanut.

Additional file 4: Table S4. FPKM values of all the genes of peanut genotypes at seedling stage.

Additional file 5: Table S5. DNA methylation pattern for the top 50 genes showing wide range of FPKM values among the peanut genotypes.

Additional file 6: Table S6. DNA methylation sites in the DNA methyl transferase and DNA demethylase genes among the peanut genotypes.

Additional file 7: Table S7a. Genes showing differential expression between the parental and its mutant progeny genotypes of peanut. Table S7b. Genes showing differential expression between the foliar disease resistant and susceptible genotypes of peanut.

Additional file 8: Table S8. Real-time PCR analysis for the foliar disease resistance-linked genes among the resistant and the susceptible genotypes of peanut.

\section{Abbreviations}

CHG: C (A, C or T) G; CpG: C phosphate G; CHH: C (A, C or T) (A, C or T); ELS: early leaf spot; LLS: late leaf spot; FPKM: fragments per kilobase of transcript per million mapped reads; QTL: quantitative trait loci; MET: methyltransferase; CMT: chromomethylase; DRM: DNA methyltransferase; qRT-PCR: quantitative reverse transcription polymerase chain reaction; PEG: polyethylene glycol; CDNA: complementary DNA; SYBR: $N^{\prime}, N^{\prime}$-dimethyl- $N$-[4-[(E)-(3-methyl-1,3benzothiazol-2-ylidene) methyl]-1-phenylquinolin-1-ium-2-yl]-N-propylpropane-1,3-diamine; G6PD: glucose-6-phosphate dehydrogenase; DME: DNA glycosylase DEMETER; QTL: quantitative trait loci; PCR: polymerase chain reaction.

\section{Acknowledgements}

We acknowledge the receipt of the genotypes from the collection of Dr. M. V. C. Gowda, Professor, Department of Genetics and Plant Breeding, University of Agricultural Sciences, Dharwad 580 005, India.

\section{Authors' contributions}

RSB: conceptualized the idea and prepared the manuscript. JR: helped in generating the methylome and transcriptome reads and analyzing the data. KS: helped in analyzing the data, submitting the sequence to the GenBank and finalizing the manuscript. IST: helped in sample collection for DNA and RNA, and carried the lab and field experiments to collect the data. MPB: helped in sample collection for DNA and RNA, and carried the lab and field experiments to collect the data. VBR: helped in generating the methylome and transcriptome reads and analyzing the data. All authors read and approved the final manuscript.

\section{Funding}

Financial support received from the Department of Science and TechnologyJapan Society for the Promotion of Science (DST-JSPS; Grant No. DST/INT/ JSPS/P-227/2016) Bilateral Program and Rashtriya Krishi Vikas Yojana (RKVY; Grant No. 2017-18) for generating the data is gratefully acknowledged.

\section{Availability of data and materials}

The sequences have been submitted, and the GenBank accession number (DRA007069) has been obtained for the methylome and transcriptome reads of GPBD 4, VG 9514, ICGV 86855, ICGV 86699, ICGV 99005, TAG 24, TMV 2, JL 24, DER, VL 1 and TMV 2-NLM). The genotypes used in this study are either the released varieties or the breeding lines.

\section{Ethics approval}

This study involves peanut varieties (publicly available and released to the farmers for cultivation) and breeding lines.

\section{Consent to participate}

Not applicable.

\section{Consent to publish}

Not applicable.

\section{Competing interests}

The authors declare that they have no competing interests.

\section{Author details}

${ }^{1}$ Department of Biotechnology, University of Agricultural Sciences, Dharwad 580 005, India. ${ }^{2}$ AgriGenome Labs Pvt. Ltd., Kochi, Kerala 682042 , India. ${ }^{3}$ Department of Frontier Research, Kazusa DNA Research Institute, Chiba 292-0818, Japan. ${ }^{4}$ AgriGenome Labs Pvt. Ltd., Hyderabad, Telangana 500078 , India.

Received: 20 July 2019 Accepted: 2 January 2020

Published online: 07 January 2020

\section{References}

1. Kolekar RM, Sukruth M, Shirasawa K, Nadaf HL, Motagi BN, Lingaraju S, et al. Marker-assisted backcrossing to develop foliar disease resistant genotypes in TMV 2 variety of peanut (Arachis hypogaea L.). Plant Breed. 2017;136(6):948-53. https://doi.org/10.1111/pbr.12549.

2. Varshney RK, Pandey MK, Pasupuleti J, Nigam SN, Sudini H, Gowda MVC, et al. Marker-assisted introgression of a QTL region to improve rust resistance in three elite and popular varieties of peanut (Arachis hypogaea L.). Theor Appl Genet. 2014;127(8):1771-81.

3. Zhuang W, Chen H, Yang M, Wang J, Pandey MK, Zhang C, et al. The genome of cultivated peanut provides insight into legume karyotypes, polyploid evolution and crop domestication. Nat Genet. 2019;51(5):865.

4. Bertioli DJ, Jenkins J, Clevenger J, Dudchenko O, Gao D, Seijo G, et al. The genome sequence of segmental allotetraploid peanut Arachis hypogaea. Nat Genet. 2019;51(5):877.

5. Murrell A, Rakyan VK, Beck S. From genome to epigenome. Hum Mol Genet. 2005;14(suppl_1):R3-10.

6. Sharma R, Vishal P, Kaul S, Dhar MK. Epiallelic changes in known stressresponsive genes under extreme drought conditions in Brassica juncea (L.) Czern. Plant Cell Rep. 2016;36(1):203-17.

7. Zhu W, Hu B, Becker C, Dogan ES, Berendzen KW, Weigel D, et al. Altered chromatin compaction and histone methylation drive non-additive gene expression in an interspecific Arabidopsis hybrid. Genome Biol. 2017;18(1):157. https://doi.org/10.1186/s13059-017-1281-4.

8. Liu S, Li F, Kong L, Sun Y, Qin L, Chen S, et al. Genetic and epigenetic changes in somatic hybrid introgression lines between wheat and tall wheatgrass. Genetics. 2015;199(4):1035-45. https://doi.org/10.1534/ genetics.114.174094.

9. Shen $S$, Wang Z, Shan X, Wang H, Li L, Lin X, et al. Alterations in DNA methylation and genome structure in two rice mutant lines induced by high pressure. Sci China C Life Sci. 2006;49(2):97-104.

10. Schmitt F, Oakeley EJ, Jost JP. Antibiotics induce genome-wide hypermethylation in cultured Nicotiana tabacum plants. J Biol Chem. 1997;272(3):1534-40.

11. Ou X, Long L, Zhang Y, Xue Y, Liu J, Lin X, et al. Spaceflight induces both transient and heritable alterations in DNA methylation and gene expression in rice (Oryza sativa L.). Mutat Res. 2009;662(1-2):44-53. https://doi. org/10.1016/j.mrfmmm.2008.12.004

12. Ossowski S, Schneeberger K, Lucas-Lledo Jl, Warthmann N, Clark RM, Shaw RG, et al. The rate and molecular spectrum of spontaneous mutations in Arabidopsis thaliana. Science. 2010;327(5961):92-4. https://doi. org/10.1126/science.1180677.

13. Miura A, Yonebayashi S, Watanabe K, Toyama T, Shimada H, Kakutani T. Mobilization of transposons by a mutation abolishing full DNA 
methylation in Arabidopsis. Nature. 2001;411(6834):212-4. https://doi. org/10.1038/35075612.

14. Ma X, Wang Q, Wang Y, Ma J, Wu N, Ni S, et al. Chromosome aberrations induced by zebularine in triticale. Genome. 2016;59(7):485-92. https:// doi.org/10.1139/gen-2016-0047.

15. Shi J, Lu W, Sun Y. Comparison of space flight and heavy ion radiation induced genomic/epigenomic mutations in rice (Oryza sativa). Life Sci Space Res. 2014;1:74-9. https://doi.org/10.1016/j.lIss.2014.02.007.

16. Zilberman D, Gehring M, Tran RK, Ballinger T, Henikoff S. Genomewide analysis of Arabidopsis thaliana DNA methylation uncovers an interdependence between methylation and transcription. Nat Genet. 2007:39(1):61-7.

17. Levy AA, Feldman M. Genetic and epigenetic reprogramming of the wheat genome upon allopolyploidization. Biol J Linn Soc Lond. 2004;82(4):607-13.

18. Zhao $X$, Chai Y, Liu B. Epigenetic inheritance and variation of DNA methylation level and pattern in maize intra-specific hybrids. Plant Sci. 2007;172(5):930-8.

19. Kour G, Kour B, Kaul S, Dhar MK. Genetic and epigenetic instability of amplification-prone sequences of a novel $B$ chromosome induced by tissue culture in Plantago lagopus L. Plant Cell Rep. 2009;28(12):1857-67. https://doi.org/10.1007/s00299-009-0789-9.

20. Gallusci P, Dai Z, Génard M, Gauffretau A, Leblanc-Fournier N, RichardMolard C, et al. Epigenetics for plant improvement: current knowledge and modeling avenues. Trends Plant Sci. 2017;22(7):610-23.

21. Wang P, Gao C, Bian X, Zhao S, Zhao C, Xia H, et al. Genome-wide identification and comparative analysis of cytosine-5 DNA methyltransferase and demethylase families in wild and cultivated peanut. Front Plant Sci. 2016:7:7. https://doi.org/10.3389/fpls.2016.00007.

22. Schubert $M$, Lindgreen $S$, Orlando L. AdapterRemoval v2: rapid adapter trimming, identification, and read merging. BMC Res Notes. 2016;9:88. https://doi.org/10.1186/s13104-016-1900-2.

23. Pedersen BS, Eyring K, De S, Yang IV, Schwartz DA. Fast and accurate alignment of long bisulfite-seq reads. arXiv preprint arXiv:14011129. 2014.
24. IPGI. High-quality genome assembly for peanut (Arachis hypogaea), cultivar "Tifrunner". 2017.

25. Akalin A, Kormaksson M, Li S, Garrett-Bakelman FE, Fiqueroa ME, Melnick $A$, et al. methylKit: a comprehensive $R$ package for the analysis of genome-wide DNA methylation profiles. Genome Biol. 2012;13(10):R87.

26. Langmead B, Salzberg SL. Fast gapped-read alignment with Bowtie 2. Nat Methods. 2012;9(4):357.

27. Dobin A, Davis CA, Schlesinger F, Drenkow J, Zaleski C, Jha S, et al. STAR: ultrafast universal RNA-seq aligner. Bioinformatics. 2013;29(1):15-21.

28. Trapnell C, Hendrickson DG, Sauvageau M, Goff L, Rinn JL, Pachter L. Differential analysis of gene regulation at transcript resolution with RNA-seq. Nat Biotechnol. 2013;31(1):46.

29. Zemach A, McDaniel IE, Silva P, Zilberman D. Genome-wide evolutionary analysis of eukaryotic DNA methylation. Science. 2010;328(5980):916-9.

30. Feng S, Cokus SJ, Zhang X, Chen P-Y, Bostick M, Goll MG, et al. Conservation and divergence of methylation patterning in plants and animals. Proc Natl Acad Sci. 2010;107(19):8689-94.

31. Vaughn MW, Tanurdžić M, Lippman Z, Jiang H, Carrasquillo R, Rabinowicz PD, et al. Epigenetic natural variation in Arabidopsis thaliana. PLoS Biol. 2007:5(7):e174

32. Suzuki MM, Bird A. DNA methylation landscapes: provocative insights from epigenomics. Nat Rev Genet. 2008;9(6):465.

33. Shirasawa K, Bhat RS, Khedikar YP, Sujay V, Kolekar RM, Yeri SB, et al. Sequencing analysis of genetic loci for resistance for late leaf spot and rust in peanut (Arachis hypogaea L.). Front Plant Sci. 2018. https://doi. org/10.3389/fpls.2018.01727.

\section{Publisher's Note}

Springer Nature remains neutral with regard to jurisdictional claims in published maps and institutional affiliations.
Ready to submit your research? Choose BMC and benefit from:

- fast, convenient online submission

- thorough peer review by experienced researchers in your field

- rapid publication on acceptance

- support for research data, including large and complex data types

- gold Open Access which fosters wider collaboration and increased citations

- maximum visibility for your research: over 100M website views per year

At BMC, research is always in progress.

Learn more biomedcentral.com/submissions 